\title{
On the role of trial outcomes in delayed discriminations
}

\author{
PETER J. URCUIOLI \\ Purdue University, West Lafáyette, Indiana \\ and \\ THOMAS R. ZENTALL \\ University of Kentucky, Lexington, Kentucky
}

\begin{abstract}
Delayed simple discriminations are typically retained more accurately over longer delays by pigeons than are delayed conditional discriminations (e.g., Honig \& Wasserman, 1981). In two experiments, we investigated the extent to which trial outcomes contribute to this difference by comparing performances when all trials ended with food reinforcement versus when only half of the trials did. Experiment 1 showed that when food was presented on all trials, contingent upon either pecking or not pecking the test stimulus, levels of retention and rates of forgetting were comparable for these two tasks. By contrast, Experiment 2 showed better retention of delayed simple than delayed conditional discriminations when half of the trials ended with food and the other half in extinction. Furthermore, delayed simple discriminations were retained more accurately with food versus no-food outcomes than with food at the end of every trial, whereas the reverse was true for delayed conditional discriminations. These findings indicate that retention differences between these tasks are another instance of the differential outcomes effect.
\end{abstract}

Honig and Wasserman (1981) and others (e.g., Cohen, Galgan, \& Fuerst, 1986; Weisman, Bruce, \& Beninger, 1987) have reported that working-memory performances are typically more accurate, and rates of forgetting slower, on delayed simple discriminations (DSDs) than on delayed conditional discriminations (DCDs). In a DSD, reinforcement for responding on the retention test is signaled solely by the stimuli presented at the beginning of the trial (i.e., the initial stimuli), whereas in a DCD, reinforcement depends jointly upon the initial stimulus and the test stimulus.

For example, in Experiment 1 of Honig and Wasserman (1981), separate groups of pigeons were trained on delayed go/no-go discriminations with red and green initial stimuli and vertical and horizontal test stimuli. For the DSD group, food was presented following the first test-stimulus response after $5 \mathrm{sec}$ on all trials beginning with one hue, but was never presented on trials beginning with the other. For the DCD group, test-stimulus responses to one line orientation were reinforced following red but not green, whereas responses to the other orientation were reinforced following green but not red. When each group was later tested with delays ranging from 1-30 sec between initial stimulus offset and test

\footnotetext{
Preparation of this manuscript was supported by NSF Grant $86-06926$ to Peter J. Urcuioli. The authors thank Nicholas Ketterer, Beth Kraemer, Judy Tedlie, and Suzanne Vanavermaete for running the subjects. Reprint requests may be addressed to Peter J. Urcuioli, Department of Psychological Sciences, Purdue University, West Lafayette, IN 47907.
}

stimulus onset, DSD birds showed higher levels of discriminative performance and slower rates of forgetting than did DCD birds.

These results are consistent with the view that subjects remember different events from trial to trial in these two tasks (i.e., the memory code differs). For instance, birds in a DSD might anticipate early in the trial whether or not they will respond to the test stimulus later in the trial. These "response intentions" (see Honig \& Dodd, 1986) could enhance retention by providing DSD birds with an additional memorial cue unavailable to birds in a DCD. Alternatively, DCD birds might also anticipate their future response patterns, although they would also have to remember to which particular test stimulus to respond or not to respond. Consequently, a difference in the complexity of the anticipatory or prospective response codes could also account for the between-group differences in retention.

Another explanation is that DSD birds anticipate not their response patterns, but whether or not food will occur at the end of a trial. Because different outcomes (food vs. no food) are typically correlated with the initial stimuli in a DSD, this correlation could give rise to differential outcome expectancies which, in turn, could provide DSD birds with an additional memorial cue. This account seems especially plausible given that learning and retention are enhanced by differential outcomes in other tasks (Carlson \& Wielkiewicz, 1976; Peterson, 1984; Trapold, 1970), and that the anticipation of those outcomes is at least partially responsible for the effect (Honig, Matheson, \& Dodd, 1984). 
In Experiment 1, as a first attempt to distinguish between the response-intention and outcome-expectancy interpretations of the DSD-DCD difference, we compared performances on these two tasks when the same outcome (food) occurred at the end of every trial. The go/no-go feature of the two tasks was retained, however, by making food contingent upon responding to the test stimulus on some trials, and upon not responding to it on other trials.

\section{EXPERIMENT 1}

In Experiment 1, two groups of pigeons were trained and tested on delayed discriminations with red and green initial stimuli and vertical and horizontal test stimuli. For both groups, reinforcement on half of the trials was contingent upon the first test-stimulus response after $5 \mathrm{sec}$. On the remaining trials, not pecking the test stimulus for $5 \mathrm{sec}$ produced food. For the DSD group, the red versus green initial stimuli signaled which pattern of test-stimulus responding would be reinforced. For the DCD group, the reinforced pattern was determined jointly by the initial and test stimuli.

If pigeons anticipate how they will respond in a DSD, and those response intentions (Honig \& Dodd, 1986) are more memorable than whatever DCD pigeons remember, then overall retention should be more accurate and the rate of forgetting slower than in a DCD. On the other hand, if the DSD-DCD difference reported by Honig and Wasserman (1981) and others (e.g., Weisman et al., 1987) arises because DSD subjects anticipate the presence versus absence of reinforcement at the end of a trial, then eliminating those differential outcomes should also eliminate the retention differences between tasks.

\section{Method \\ Subjects. Eight experimentally naive White Carneaux pigeons (retired breeders) obtained from the Palmetto Pigeon Plant (Sum- ter, SC) served as subjects. They were housed individually in stain- less steel, wire-mesh cages in a colony room with a $14: 10 \mathrm{~h}$ light:dark cycle and were maintained at $80 \%$ of their free-feeding body weights during the experiment by restricted feeding. Grit and water were freely available in the home cage. The birds received their entire daily ration of mixed grain in the experimental appara- tus, except for 1 day each weekend when they were fed in the home} cage.

Apparatus. A standard BRS/LVE pigeon chamber equipped with a three-key intelligence panel (Model PIP-016) was used for the experiment. The three horizontally aligned clear pecking keys were mounted behind circular holes, $2.5 \mathrm{~cm}$ in diameter, located $25.5 \mathrm{~cm}$ from the chamber floor and spaced $8.3 \mathrm{~cm}$ center to center. Only the center key was used in this experiment. An inline projector unit (Model IC-901-IDD) mounted directly behind the center key projected red (R), green (G), and white (W) homogeneous fields and a single white vertical (V) or horizontal (H) line on a black background (Pattern No. 696). The $5.0 \times 5.8 \mathrm{~cm}$ opening for the rear-mounted grain magazine was centered $13 \mathrm{~cm}$ below the center key and $12 \mathrm{~cm}$ above the grid floor. General chamber illumination was provided by a partially covered GE No. 1829 bulb, $7.6 \mathrm{~cm}$ above the center key, which directed light toward the ceiling. A blower fan mounted on the outside of the chamber ran continuously to mask extraneous noises. Experimental events were controlled and monitored by a Cromemco Z-2D microcomputer.

Procedure. After initial training to eat out of the food hopper and to peck hues and lines on the center response key, all birds received seven sessions of training during which pecking $V$ and $\mathrm{H}$ lines (Sessions $!-2$ ) or $R$ and $G$ hues (Sessions 3-7) was reinforced on modified fixed-interval (FI) schedules. In the first session with each pair of stimuli, food was contingent upon the first peck after $2 \mathrm{sec}$, timed from the initial peck to the stimulus for that trial (an FI 2-sec schedule). In the second session with the lines and the second and third sessions with the hues, reinforcement was delivered on an FI 5-sec schedule. In the fourth and fifth sessions with the hues, the FI value was $10 \mathrm{sec}$. Each line or hue stimulus was presented 30 times in each session with the restriction that neither stimulus could appear more than three times in succession. The duration of food reinforcement was constant within a session for individual subjects but varied between 2 and $5 \mathrm{sec}$ across sessions in order to maintain body weights as close to $80 \%$ of their freefeeding values as possible. Successive trials were separated by a 10-sec intertrial interval (ITI). The houselight was off during all but the last $1 \mathrm{sec}$ of the ITI, at which time it was turned on for the duration of the trial.

The birds then began training on either simple or conditional go/no-go discriminations with $R$ and $G$ initial stimuli and $V$ and H test stimuli (cf. Honig \& Wasserman, 1981). Trials began with a $10-\mathrm{sec}$ presentation of one of the two hues, timed from the initial peck to that stimulus. The first peck after $10 \mathrm{sec}$ turned the initial stimulus off, initiated a blank 0.5 -sec interstimulus interval, and then produced one of the two line orientations as the test stimulus. For half of the birds in Group SD, the first keypeck to either $V$ or $\mathrm{H}$ after $5 \mathrm{sec}$ produced food if the initial stimulus had been $\mathrm{R}$, whereas not pecking either test stimulus for $5 \mathrm{sec}$ produced food if the initial stimulus had been $\mathbf{G}$ (a differential reinforcement of other behavior, or DRO, 5-sec schedule). These contingencies were reversed for the remaining $2 \mathrm{SD}$ birds. For half of the birds in Group $C D$, pecking the $V$ test stimulus and not pecking the $H$ test stimulus were reinforced following $R$, whereas pecking $H$ and not pecking $\mathrm{V}$ were reinforced following $\mathrm{G}$. The remaining $\mathrm{CD}$ birds had the opposite contingencies. For both groups, pecking the test stimulus on DRO trials simply delayed reinforcement by resetting the DRO interval. The four trial types for each group are shown in the top row of Table 1

Table 1

Treatment Conditions for Experiment 1 and Experiment 2

\begin{tabular}{ll}
\multicolumn{2}{c}{ Group SD } \\
\hline$R \rightarrow V$ & FI $5 \mathrm{sec}$ \\
$R \rightarrow H$ & FI $5 \mathrm{sec}$ \\
$G \rightarrow V$ & DRO $5 \mathrm{sec}$ \\
$G \rightarrow H$ & DRO $5 \mathrm{sec}$
\end{tabular}

\begin{tabular}{ll}
\multicolumn{2}{c}{ Group CD } \\
\hline$R \rightarrow V$ & FI $5 \mathrm{sec}$ \\
$R \rightarrow H$ & DRO $5 \mathrm{sec}$ \\
$G \rightarrow V$ & DRO $5 \mathrm{sec}$ \\
$G \rightarrow H$ & FI $5 \mathrm{sec}$
\end{tabular}

\begin{tabular}{ll}
\multicolumn{2}{c}{ Group SE } \\
\hline$R \rightarrow V$ FI 5 sec \\
$R \rightarrow H$ FI 5 sec \\
$G \rightarrow V$ EXT
\end{tabular}

\begin{tabular}{ll}
\multicolumn{2}{r}{ Group CE } \\
\hline$R \rightarrow V$ & FI 5 sec \\
$R \rightarrow H$ & EXT \\
$G \rightarrow V$ & EXT \\
$G \rightarrow H$ & FI 5 sec
\end{tabular}

Note $-\mathrm{R}=$ red homogeneous field, $\mathrm{G}=$ green homogeneous field, $\mathrm{V}=$ vertical white line on black background, $\mathrm{H}=$ horizontal white line on black background, $\mathrm{Fl}=$ fixed-interval schedule, $\mathrm{DRO}=$ differential reinforcement of other behavior schedule, EXT = extinction. Initial stimulus contingencies and counterbalancing of the end-of-trial reinforcement schedules are omitted for clarity. 
Training sessions consisted of 60 delayed discrimination trials, 15 with each combination of initial and test stimulus, and 10 additional trials on which food was presented immediately following the initial stimulus. The latter were included in order to maintain approximately equivalent rates of responding to the initial stimuli (cf. Honig \& Wasserman, 1981). A 10-sec ITI separated successive trials. The houselight was turned on for the last $1 \mathrm{sec}$ of the ITI and remained on until the end of a trial. Reinforcement duration was again adjusted on a session-by-session basis for each bird in order to maintain $80 \%$ body weights as closely as possible.

With the exception of one Group CD bird (CD1), the subjects were trained on their respective tasks until they reached a criterion of five of six consecutive sessions with $90 \%$ or more of their teststimulus responses occurring on trials on which pecking the test stimulus was reinforced. Once criterion was reached, the birds began retention testing with three different delays between the initial and test stimuli randomly scheduled in each session. These delays were 0,5 , and $10 \mathrm{sec}$ for the first 20 test sessions ("short delays"), 0,10 , and $20 \mathrm{sec}$ for the next 20 sessions ("long delays"), and 0,5 , and $10 \mathrm{sec}$ again for the final 20 sessions. Each delay within a set occurred five times with each combination of initial and test stimuli. Otherwise, all procedural details were identical to those for acquisition. Testing for Bird CD1 began after 70 sessions even though it had not yet reached the $90 \%$ criterion. Nevertheless, its consistently high level of discriminative performance (i.e., discrimination ratios between .81 and .89 ) was judged sufficient enough to proceed on to testing.

Data analysis. Discrimination ratios (DRs), calculated by dividing the total number of test-stimulus responses on trials on which pecking was reinforced by the total number of test-stimulus responses across all trials, were used to assess discriminative performance. In order to equate for the opportunity to peck the test stimulus on both DRO trials and trials on which pecking was reinforced, only responses occurring during the first $5 \mathrm{sec}$ of each test stimulus entered into the computations. For the statistical analyses of the DRs and other data, Type I error rate was set at .05 and was controlled on a perdecision basis by using the tabled values provided by Rodger (1975a).

\section{Results}

Figure 1 shows acquisition of the simple and conditional discriminations over the first 16 sessions of training. Both groups initially responded nondifferentially to the test stimuli but, as training progressed, performances became more and more discriminative. Furthermore, the rate of acquisition was considerably faster in Group SD than in Group CD. Analysis of variance (ANOVA) revealed significant effects of group $[F(1,6)=57.18]$ and session $[F(14,84)=13.87]$, and a group $\times$ session interaction $[F(14,84)=3.20]$. The difference in acquisition was also apparent in the average number of sessions for each group to reach a particular performance level. For example, Group SD required only 6.5 sessions to achieve a $.80 \mathrm{DR}$, whereas Group CD needed 28.8 sessions $[F(1,6)=6.52]$.

Figure 1 also shows DRs for initial-stimulus responding in Group SD. Since the $R$ and $G$ hues were correlated with go versus no-go patterns of test-stimulus responding in this group, this correlation might induce similar differential responding to the initial stimuli which, in turn, could facilitate acquisition (Urcuioli \& Honig, 1980). In fact, the SD birds did respond differentially to the initial stimuli early in training, but by the seventh session the birds were responding about equally often to each hue. Thus, any influence of differential initial-stimulus responding on acquisition in this group appeared to be small and restricted largely to the early sessions of training.

Figure 2 shows how responding on the go and no-go trials changed over the course of acquisition. Both groups responded more frequently on go trials, and less frequently on no-go trials over the last five than over the first five sessions of acquisition. Both of these differences were significant in Group SD $\left[F_{\mathrm{S}}(1,3)=28.6\right.$ and 226.0 , respectively]. In Group $\mathrm{CD}$, the change on no-go trials was significant $[F(1,3)=46.77]$, but was not on go trials $[F(1,3)$ $=2.23]$. The reason for the latter result was that 1 of the 4 Group CD birds pecked the test stimulus on go trials less often at the end than at the beginning of acquisition.

Figure 3 plots each group's retention function averaged over the 20 sessions with each set of delays. The two

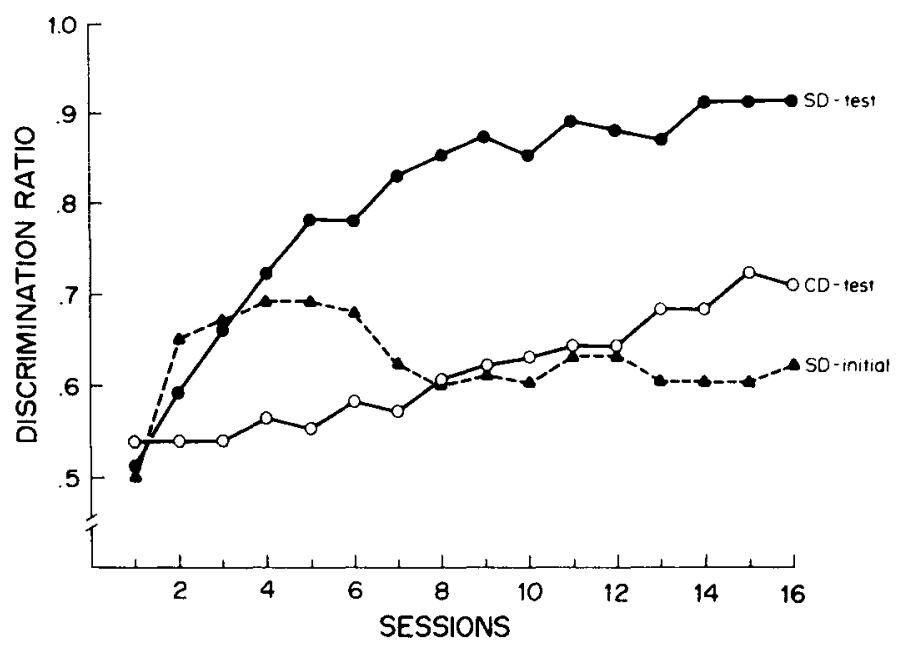

Figure 1. Mean discrimination ratios for test-stimulus responding during acquisition by the simple (SD-test) and conditional discrimination (CD-test) groups in Experiment 1, and for initial-stimulus responding by the simple discrimination group (SD-initial). 


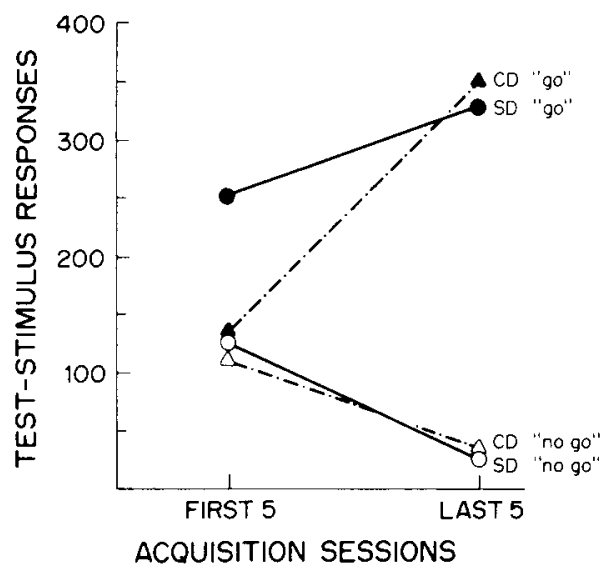

Figure 2. Mean number of test-stimulus responses on go and nogo trials for each group over the first and last five acquisition sessions of Experiment 1.

groups performed very similarly on the first short-delay test (left panel, solid lines). Furthermore, retention interval had very little effect on performance in either group. These impressions were confirmed by ANOVA, which showed no between-group difference in overall performance $[F(1,6)=0.51]$, no effect of delay $[F(2,12)=$ $2.12]$, and no group $\times$ delay interaction $[F(2,12)=0.52]$.

With the longer set of delays (right panel), average performance was somewhat better in Group SD than in Group $C D$, with the largest difference appearing at the 0 -sec value. Again, however, discriminative performances showed little change with increasing retention interval. Even at the 20 -sec delay, $70 \%-75 \%$ of all test-stimulus responses continued to occur on go trials. ANOVA on the long-delay data showed that the overall effect of delay was significant $[F(2,12)=22.65]$, but neither the group effect nor the group $\times$ delay interaction were $[F(1,6)=2.28$ and $F(2,12)=1.74$, respectively $]$.
On the second short-delay test (left panel, dashed lines), DRs were higher at every retention interval in Group SD than in Group CD. This difference also appeared to be slightly larger at the 5- and 10-sec delays than at $0 \mathrm{sec}$. ANOVA confirmed Group SD's superior overall retention $[F(1,6)=7.29]$, and the overall effect of delay $[F(2,12)=4.54]$. The group $\times$ delay interaction was not significant, however $[F(2,12)=1.01]$.

\section{Discussion}

The major finding of this experiment was that pigeons' retention of delayed simple and delayed conditional discriminations is comparable (cf. Honig \& Wasserman, 1981) when food reinforcement occurs at the end of every trial. Group SD's performance on the first two retention tests was statistically indistinguishable from that of Group $\mathrm{CD}$, despite the fact that the simple discrimination was acquired more quickly than the conditional discrimination. Although the absence of retention differences might be attributed to the relatively small number of subjects per group, exactly the same number of subjects per group was sufficient to yield the large differences between simple and conditional discriminations reported by Honig and Wasserman (1981). Thus, the present findings indicate that the superiority of DSDs over DCDs is related to the use of food versus no-food outcomes at the end of a trial. When all trials end in food, that superiority greatly diminishes.

Still, Group SD did respond more discriminatively than Group CD on the second short-delay test. But despite its advantage in overall level of retention, Group SD showed the same rate of forgetting as did Group CD. This suggests that the birds in both groups might have been retaining the same within-trial events (e.g., the initial stimuli), but that those events were more strongly encoded in Group SD than in Group CD (Honig \& Thompson, 1982). Alternatively, the results from the second test could

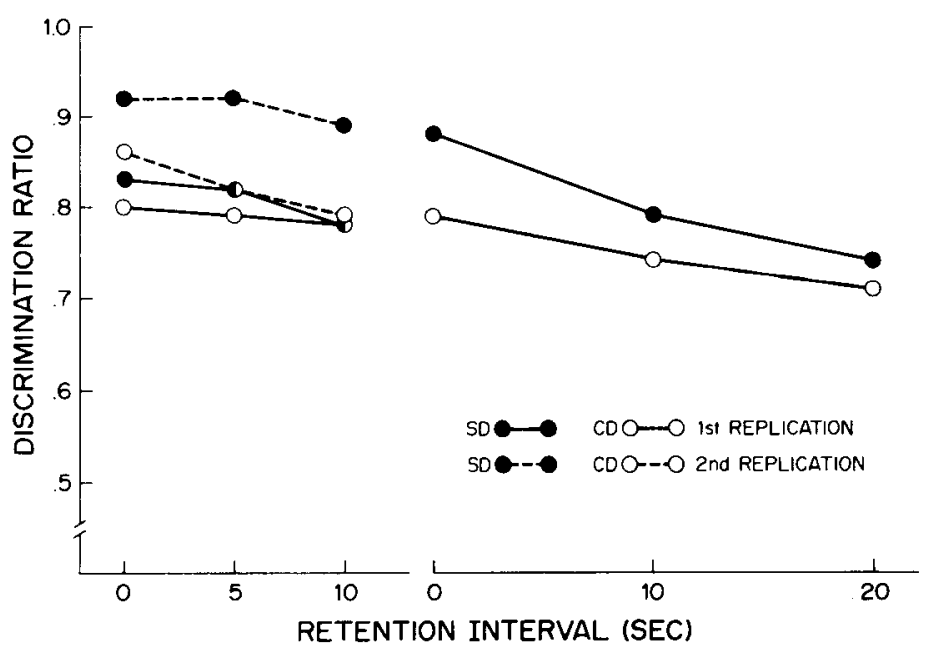

Figure 3. Mean discrimination ratios by delay for the simple (SD) and conditional discrimination (CD) groups during retention testing in Experiment 1. 
mean that the Group SD birds had learned to anticipate their go versus no-go response patterns over the course of repeated testing. Still, when viewed in comparison with Honig and Wasserman's (1981) findings, the data suggest that response intentions (if involved) have at best only a minor role in producing the retention differences between simple and conditional discriminations. By implication, then, different trial outcomes and the resultant possibility of their anticipation in simple discriminations are apparently responsible for the DSD-DCD difference. This implication was directly tested in the next experiment.

\section{EXPERIMENT 2}

In Experiment 2, we followed up on the results of Experiment 1 by comparing DSDs and DCDs when trial outcomes differed from trial to trial versus when they were always the same. Thus, an attempt was made to replicate the results of the preceding experiment and to see whether or not retention differences between DSDs and DCDs could be "recovered"' if half the trials ended with no food (i.e., in extinction; cf. Honig \& Wasserman, 1981; Weisman et al., 1987). The $2 \times 2$ (task $\times$ outcome) design also permitted an assessment of the role of trial outcomes within each task. For example, retention of DSDs might be enhanced by food versus no-food outcomes if the correlation of outcomes with the initial stimuli make the latter more distinctive, or if it gives rise to an additional (outcome-expectancy) cue. By contrast, food versus nofood outcomes would not be expected to enhance performance of DCDs because the conditional relationship between initial and test stimuli ensures that those outcomes will occur equally often following each initial stimulus.

\section{Method}

Subjects and Apparatus. Sixteen experimentally naive White Carneaux pigeons obtained from the Palmetto Pigeon Plant (Sumter, SC) served in this experiment. All were retired breeders and were housed in the same fashion as the birds in Experiment 1. The present experiment was run in two replications of 8 birds each. In each replication, the 8 subjects were randomly assigned to one of four groups prior to the start of the experiment. One DSD subject was replaced during the acquisition phase of the second replication when it was discovered that it was being run incorrectly. A DCD subject from the same replication was dropped from the experiment after 70 sessions of acquisition because it was unable to maintain consistently high levels of discrimination performance.

The apparatus and stimuli were identical to those used in Experiment 1.

Procedure. Initial training to eat out of the food hopper and to peck hues and lines on the center key was identical to that described in Experiment 1. All birds then received two sessions of reinforced pecking to $\mathrm{V}$ and $\mathrm{H}$ center-key lines with food delivered on modified FI schedules like those described in Experiment 1. For birds assigned to Group SD and Group CD (see below), these sessions were identical to those in Experiment 1. For birds assigned to Group SE and Group CE (see below), however, only half of the 30 trials with each line stimulus ended with food reinforcement. On the remaining trials, the line stimulus was simply turned off and the ITI began immediately following the end of the FI. Partial reinforcement was scheduled during this phase for Group SE and Group CE in order to be consistent with partial reinforcement for test-stimulus responding these birds would later experience during training on their respective go/no-go discriminations.

Next, the birds in all four groups received five sessions of reinforced training for pecking $R$ and $G$ center-key hues. These sessions were identical in every respect to those described in Experiment 1 , with each trial ending in food reinforcement for all groups.

Delayed discrimination acquisition with $R$ and $G$ initial stimuli and $\mathrm{V}$ and $\mathrm{H}$ test stimuli began following the pretraining phases described above. Group SD and Group CD were treated in the same fashion as the corresponding groups in Experiment 1. For them, food reinforcement occurred at the end of each trial contingent upon either responding or not responding to the test stimulus. For Group SE and Group CE, on the other hand, half of the trials ended in extinction rather than in the DRO contingency. On EXT trials, the test stimulus simply went off after $5 \mathrm{sec}$. In Group SE, the initial stimuli alone signaled whether or not responding would be reinforced at the end of a trial. In Group CE, both the initial and test stimuli were necessary to make that determination. The contingencies for all four groups are summarized in Table 1.

As in Experiment 1, each bird was run on its respective task until it achieved a DR of 90 or better for five of six consecutive sessions. Then it was tested for 20 sessions with 0-, 5-, and 10-sec delays (short delays) between the initial and test stimuli, followed by 20 sessions with $0-, 10-$, and 20 -sec delays (long delays), and finally by 10 additional sessions with short delays. In all of these sessions, delays occurred randomly across trials with the constraint that each delay occurred equally often with each of the four trial types. Refresher sessions on the acquisition task (viz. with 0 -sec delays only) were periodically given during testing if the birds could not maintain a high level of 0 -sec delay performance. Refresher sessions were very infrequent, were generally confined to the early stages of testing, and were continued until DRs rose above .85 .

\section{Results}

Table 2 summarizes the acquisition results in terms of the number of sessions required by each group to reach DRs of .80 and .90 . By either criterion, the DSD groups (SE and SD) acquired their go/no-go discriminations faster than the DCD groups (CE and $C D$ ), and Group $C D$ learned more quickly than Group CE. The two DSD groups showed identical rates of acquisition to a $.80 \mathrm{cri}^{-}$ terion, but Group SE appeared to learn faster than Group SD to a .90 criterion.

Statistical analyses of both sets of data revealed exactly the same pattern. Groups SE and SD did not differ in the number of sessions to reach either level of performance [both $F \mathrm{~s}(3,11)<0.10$ ], but required significantly fewer sessions of training on average than did Group CE $[F \mathrm{~s}(3,11)=12.44$ and 2.90 , respectively $]$. The number of sessions to criterion for Group CD was intermediate between the DSD groups and Group CE [both $F_{\mathrm{S}}(3,11)$ $=0.00$ ]. These results imply that acquisition by either criterion was ordered from fastest to slowest as follows:

\section{Table 2}

Average Number of Sessions to Reach a Discrimination Ratio (DR) of .80 and .90 for Each Group in Experiment 2

\begin{tabular}{crr} 
& \multicolumn{2}{c}{ DR } \\
\cline { 2 - 3 } Group & .80 & .90 \\
\hline SD & 5.5 & 12.0 \\
SE & 5.5 & 8.5 \\
CD & 8.7 & 16.0 \\
CE & 14.5 & 28.0 \\
\hline
\end{tabular}




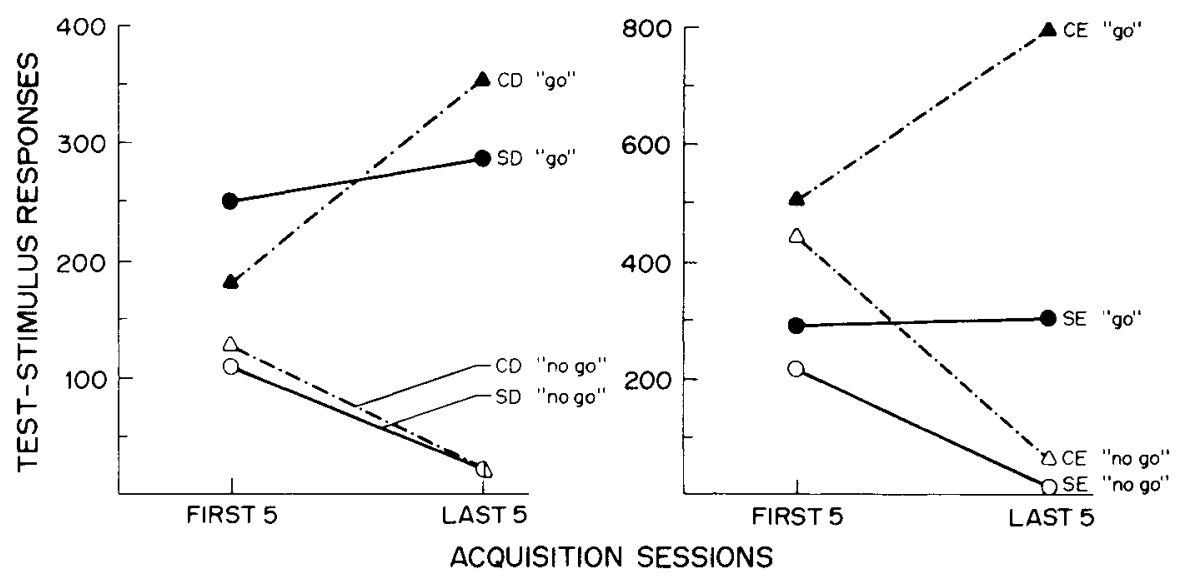

Figure 4. Mean number of test-stimulus responses on go and no-go trials for the DRO groups (left panel) and the EXT groups (right panel) over the first and last five acquisition sessions of Experiment 1 .

\section{Group SE $=$ Group SD $>$ Group $\mathrm{CD}>$ Group CE} (Rodger, 1975b).

Figure 4 shows how responding on go and no-go trials in each group changed over the course of acquisition. Data for the groups with the DRO and EXT contingencies are shown in the left and right panels, respectively. Note that the ordinate scale for the EXT condition is twice that of the DRO condition. ${ }^{1}$ All four groups responded less often to the test stimuli on no-go trials over the last five than over the first five sessions of acquisition (all $F \mathrm{~s}>13.3$ ). Responding on go trials increased over the course of acquisition in the DCD groups $[F(1,2)=26.60$ for Group $\mathrm{CD}$, and $F(1,3)=75.00$ for Group CE], but did not change significantly in either DSD group $[F \mathrm{~s}(1,3)=$ 0.52 and 0.03 for Groups SD and SE, respectively].

Figure 5 shows the retention data from the first shortdelay test and the long-delay test that immediately followed it. The data from both tests reveal exactly the same pattern of findings. Group SE exhibited the highest DRs over all delays, and showed very little drop in performance with increasing retention intervals. At the other extreme was Group CE. Retention in this group was clearly inferior to that of the other three groups, and it showed the largest drop in DRs with increasing delays. Groups SD and CD performed at an intermediate level. The latter groups maintained DRs of .70 or better at retention intervals as long as $20 \mathrm{sec}$, but their performances at these longer delays were noticeably poorer than at the shorter ones. Also, as in Experiment 1, overall levels of retention and rates of forgetting in these two groups were similar.

ANOVA on each data set revealed significant effects of group $[F \mathrm{~s}(3,11)=16.72$ and 10.73$]$ and delay $\left[F_{\mathrm{s}}(2,22)=38.76\right.$ and 68.43$]$, and a group $\times$ delay interaction $\left[F_{\mathrm{s}}(6,22)=8.40\right.$ and 5.21 for short and long delays, respectively]. Post hoc contrasts (Rodger, 1975b) across the sample means were then used to specify the between-group differences at each point along the gra-

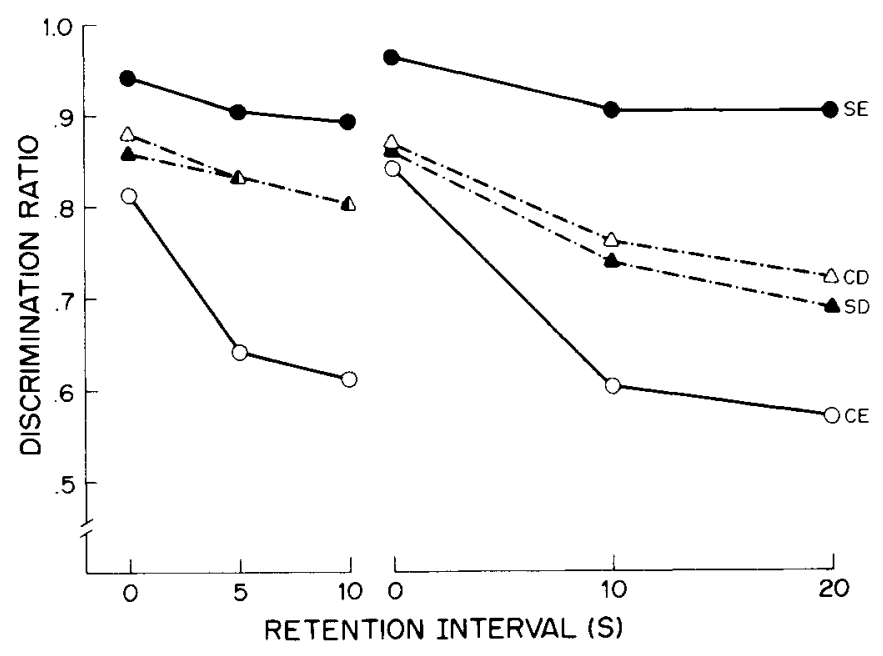

Figure 5. Mean discrimination ratios by delay for the simple and conditional discrimination groups during retention testing in Experiment 2. 
dients. Group SD did not differ from Group CD at any retention interval [all $F_{\mathrm{s}}(3,11)<0.10$ ]. By contrast, Group SE performed at higher levels of discrimination accuracy than did Group CE at all retention intervals [all $F \mathrm{~s}(3,11)>3.35$ ]. Finally, the level of performance in Groups SD and CD generally fell midway between the corresponding levels for Groups SE and CE [all $\left.F_{\mathrm{s}}(3,11)<1.46\right]$. Thus, the results from the post hoc analyses indicate that discriminative performances were consistently ordered from highest to lowest in the following fashion: Group SE > Group SD = Group CD > Group CE.

Following the last session with the long-delay set, all birds were retested for 10 sessions with the short delays. In all four groups, the level of discrimination performance increased over that observed during the initial short-delay tests, but the between-group differences in performance remained the same.

Tables 3 and 4 present the mean response rates to the initial stimuli and during the retention intervals, respectively, for individual birds in each group on short- and long-delay tests. Table 3 shows that most birds responded at approximately the same rate to the initial stimuli, as would be expected given that responding to each was intermittently reinforced in each session. The only exceptions were Birds SE1 and SE5: they responded less often to $R$ than to $G$. Interestingly, the R stimulus for both of these birds signaled end-of-trial reinforcement for responding to the test stimuli. The only other effect of note was that, on average, response rates were higher in Group CE than in the other groups.

Table 4 shows that responding during the delay interval was very infrequent in Groups SD and $C D$. By contrast, the Group CE birds frequently pecked the dark center key during the delays, albeit nondifferentially following $R$ and G. Rates in Group SE fell in between, with 2 birds (SE1 and SE2) responding more during the delay that preceded end-of-trial reinforcement.

\section{Discussion}

The results of Experiment 2 replicate the major finding of Experiment 1, that delayed simple and delayed conditional discriminations are retained equally well at delays as long as $20 \mathrm{sec}$ when each trial ends with food reinforcement. Experiment 2 also showed, however, that retention was substantially better on DSDs than on DCDs when half of the trials ended with no food. The latter result confirms the difference between these tasks reported by Honig and Wasserman (1981), and also indicates that the absence of corresponding differences when outcomes are the same on every trial is not simply due to small sample sizes.

Instead, the pattern of results shows that different trial outcomes (food vs. no food) and their correlation with the initial stimuli in a DSD play a major role in producing the relatively good retention seen in this task. Specifically, the level of discrimination performance by Group SE on both the short- and the long-delay tests was considerably higher than that observed in Group CE, whereas when all trials ended with food, retention was no better on the DSD (Group SD) than on the DCD (Group CD). Differential outcomes also enhanced DSD performance (Group SE) relative to nondifferential outcomes (Group SD). Finally, these between-group differences did not appear to be related in any logical way to differences in initial-stimulus responding or to differences in the rate or patterning of delay-interval responding.

Interestingly, the difference between the effects of the outcome conditions was exactly the opposite in the two tasks. In the DSDs, food versus no-food outcomes produced better overall retention and slower rates of forgetting than when food occurred at the end of every trial. In the DCDs, on the other hand, poorer retention was observed in the former than in the latter condition. The retention differences between the two simple discriminations can be explained by the presence versus absence of outcome expectancies in Groups SE and SD, respectively.

Table 3

Mean Response Rates (pecks/sec) to the Red (R) and Green (G) Initial Stimuli During the Short- and Long-Delay Tests of Experiment 2

\begin{tabular}{|c|c|c|c|c|c|c|c|c|c|c|c|c|c|c|c|}
\hline \multirow{2}{*}{$\begin{array}{c}\text { Stimulus- } \\
\text { Delay }\end{array}$} & \multicolumn{15}{|c|}{ Bird } \\
\hline & SD5 & SD6 & SD7 & SD8 & SE1 & SE2 & SEA & SE5 & CD5 & CD6 & CD7 & CEl & CE2 & CE3 & CE4 \\
\hline Red-Short & 1.65 & 0.92 & 1.50 & 1.63 & 1.76 & 2.34 & 2.90 & 1.20 & 1.38 & 2.03 & 0.99 & 4.84 & 2.18 & 2.76 & 3.08 \\
\hline Red-Long & 1.96 & 1.25 & 2.02 & 2.18 & 1.74 & 2.80 & 3.22 & 1.68 & 1.59 & 2.52 & 1.29 & 4.60 & 2.85 & 2.70 & 3.14 \\
\hline Green-Short & 1.53 & 1.03 & 1.38 & 1.60 & 2.22 & 2.29 & 2.60 & 2.48 & 1.02 & 1.57 & 0.96 & 4.54 & 2.47 & 2.69 & 3.16 \\
\hline Green-Long & 1.89 & 1.23 & 1.90 & 2.07 & 2.85 & 2.67 & 3.61 & 2.54 & 1.36 & 2.28 & 1.23 & 4.44 & 2.49 & 2.72 & 3.13 \\
\hline
\end{tabular}

Table 4

Mean Delay-Interval Response Rates (pecks/sec) After the Red (R) and Green (G) Initial Stimuli for the Short- and Long-Delay Tests of Experiment 2

\begin{tabular}{|c|c|c|c|c|c|c|c|c|c|c|c|c|c|c|c|}
\hline \multirow{2}{*}{$\begin{array}{c}\text { Stimulus- } \\
\text { Delay }\end{array}$} & \multicolumn{15}{|c|}{ Bird } \\
\hline & SD5 & SD6 & SD7 & SD8 & SE1 & SE2 & SE4 & SE5 & CD5 & CD6 & $\mathrm{CD} 7$ & CE1 & $\mathrm{CE} 2$ & CE3 & CEA \\
\hline Red-Short & 0.46 & 0.18 & 0.10 & 0.39 & 1.38 & 0.14 & 0.42 & 0.30 & 0.01 & 0.05 & 0.12 & 1.38 & 1.76 & 0.90 & 2.67 \\
\hline Red-Long & 0.16 & 0.08 & 0.04 & 0.20 & 1.66 & 0.14 & 0.06 & 0.12 & 0.00 & 0.06 & 0.05 & 1.70 & 1.62 & 0.60 & 2.80 \\
\hline Green-Short & 0.14 & 0.16 & 0.02 & 0.45 & 0.16 & 0.90 & 0.78 & 0.08 & 0.00 & 0.04 & 0.10 & 1.06 & 1.80 & 0.98 & 2.66 \\
\hline Green-Long & 0.14 & 0.06 & 0.02 & 0.16 & 0.04 & 1.34 & 0.42 & 0.02 & 0.00 & 0.06 & 0.04 & 1.71 & 1.54 & 0.52 & 2.79 \\
\hline
\end{tabular}


When different outcomes are correlated with the initial stimuli, birds potentially have another memorial cue (viz. an outcome expectancy) besides that provided by the initial stimuli and/or by response anticipations. That additional cue is likely to be sufficient to enhance performance (Honig et al., 1984).

The effect in the conditional discrimination groups, however, cannot be explained in an analogous fashion. For one, the initial stimuli in Group CE were equally often associated with the food and the no-food outcomes, thus precluding the development of differential outcome expectancies prior to the retention interval. For another, the difference between the DSDs was not simply absent in the conditional discrimination tasks, but was reversed. Thus, other factors must have been involved in producing the retention differences between Group CE and Group CD.

One possibility is that the DRO contingency in Group CD was more effective in suppressing responding on no-go trials than was the EXT contingency in Group CE. After all, pecking the test stimulus on DRO trials was penalized by delaying food reinforcement and the advance to the next trial. No such "penalties" occurred for responding on the corresponding EXT trials in Group $C E$ which, interestingly enough, also responded more than any other group during the retention intervals. Another explanation is that correctly remembering whatever it is that DCD birds remember was reinforced more often in Group CD than in Group CE, given that food occurred on every trial in the former group but on only half of the trials in the latter.

Of course, the same reasoning could also be applied to the DSD tasks (Group SE vs. Group SD), yet the results were opposite in direction. This does not mean, however, that the above analyses are incorrect. Rather, it may only mean that the influence of reinforcement factors on retention performances in go/no-go tasks is apparent only when the possibility of outcome expectancies based on those end-of-trial reinforcers is eliminated. Otherwise, the anticipation of the reinforcing event itself may provide such a distinctive memorial cue that it masks whatever other influence the reinforcer has on end-of-trial responding.

\section{GENERAL DISCUSSION}

The results of the experiments reported here suggest that differential outcome expectancies are responsible for the differences in retention between delayed simple and conditional go/no-go discriminations (Honig \& Wasserman, 1981). The correlation of different trial outcomes (food vs. no food) with the initial stimuli facilitated memory performance relative to tasks in which that correlation was absent (e.g., the conditional discriminations). By contrast, the anticipation of go versus no-go responding to the test stimuli (response intentions) by DSD subjects does not appear to contribute in any substantive way to the DSD-DCD effect. After all, the birds in Group SD could presumably have anticipated their test-stimulus response patterns, yet their retention was no better than that of the birds that could not have anticipated those patterns (i.e., Group CD).

Differences in task complexity (e.g., simple discriminations are "easier" than conditional discriminations) will also not account for the results (cf. Honig \& Dodd, 1983), as shown by the dissociations between rates of initial acquisition and retention. For example, Group SD acquired its discrimination faster than did Group CD in both Experiments 1 and 2 , yet retention performances in these two groups were virtually equivalent. Also, Groups SE and SD in Experiment 2 learned their respective simple discriminations at roughly the same rate, yet Group SE's retention performance was far superior to that of Group SD.

These dissociations are understandable on the assumption that the processes involved in acquisition (i.e., in the development of "reference memory"; Honig, 1978) differ in some respects from those operating once acquisition is complete (i.e., after reference memory has been established). For instance, the birds in the conditional tasks had to learn more component discriminations than did those in the simple tasks. In particular, the DCD birds had to distinguish between the $\mathrm{V}$ and $\mathrm{H}$ test stimuli in order to respond appropriately to them, whereas the DSD birds did not. That difference alone, coupled with the fact that line discriminations are relatively difficult for pigeons (Carter \& Eckerman, 1975), may explain why the two tasks were learned at different rates. Once these discriminations were learned, however, retention tests assessed which of a variety of factors might mediate workingmemory performance.

The results of those tests showed, among other things, that retention differences between tasks were not attributable in any straightforward way to either the rate or the patterning of initial-stimulus responding. For example, overall response rates to the initial stimuli during testing in Experiment 2 were highest on average in the group showing the poorest retention (Group CE). If the level of retention simply reflected the degree of attention to the initial stimuli, and the latter were appropriately indexed by how much the birds pecked at those stimuli, then Group CE should have shown the best retention, not the worst. Furthermore, if the birds differed only in how strongly they had encoded the initial stimuli, then rates of forgetting should have been comparable across groups, not different as was found in Experiment 2 (Honig \& Thompson, 1982).

There was also very little evidence that differential initial-stimulus responding contributed to the effects. The intermittent reinforcement for pecking $R$ and $G$ generated roughly equivalent rates of responding to these stimuli in all groups. Furthermore, the differential responding that was observed in Group SE of Experiment 2 was minor and unrelated to the level of retention. Consequently, although differential sample behaviors are known to enhance learning and retention (Hogan, Pace, \& Zentall, 
1983; Urcuioli, 1985; Urcuioli \& Honig, 1980), they do not seem to be involved here (see also Honig \& Wasserman, 1981).

Another factor that is more difficult to discount is differential delay-interval responding, which is known to contribute significantly to memory performances in other paradigms (Blough, 1959; Zentall, Hogan, Howard, \& Moore, 1978), and was most common in the present study in the group showing the best retention (Group SE). Again, however, this factor alone does not appear to provide a sufficient explanation for Group SE's superior performance given that the SE birds that showed little if any differential delay-interval pecking performed as well as those that showed a large amount. Besides, it is not clear whether such differential behavior produces better performance on the retention test or is simply another effect of the "true" controlling variable.

It is clear, however, that the primary controlling variable was not the anticipation of go/no-go responding. Rather, the results suggest that the anticipation of different trial outcomes (outcome expectancies) enhanced Group SE's memory performance. The correlation of the initial stimuli with food versus no food at the end of the trial produced the highest overall level of retention and virtually no loss of discriminative performance with delays as long as $20 \mathrm{sec}$. This enhanced performance is similar to what previous researchers have observed in other tasks involving different outcomes that are uniquely associated with stimuli presented at the beginning of a trial (Brodigan \& Peterson, 1976; DeLong \& Wasserman, 1981). Since that association is known to be sufficient to enhance memory performance (Honig et al., 1984), the suggestion is that outcome expectancies underlie the enhanced performance in Group SE seen here and the differences between simple and conditional discrimination tasks reported by others (Honig \& Wasserman, 1981; Weisman et al., 1987).

Cohen et al. (1986) have reported results consistent with this interpretation. Using a within-subjects design, they found (in their Experiment 2) that rats did not retain DSDs any more accurately on average than DCDs when reinforcement could be obtained at the end of every trial. They also found (in their Experiment 1) better retention of DSDs with "asymmetrical reinforcement" (i.e., differential outcomes) than with "symmetrical reinforcement" (i.e., nondifferential outcomes). As in our experiments, Cohen et al. did not reinforce responding to produce symmetrical reinforcement although, unlike the present procedure, reinforcement was omitted on those trials if the rats responded (J. Cohen, personal communication, 1988). Nonetheless, this contingency would still reduce the correlation between the initial stimuli and trial outcomes relative to the asymmetrical condition. Consequently, their data also support our analyses.

Interestingly, the conclusion that outcome expectancies, and not response anticipations, are responsible for the DSD-DCD retention difference differs from that reached by Pontecorvo (1985) on the basis of a study similar in purpose to the present experiments. Pontecorvo trained separate groups of pigeons on delayed-pair comparison procedures (Shimp \& Moffitt, 1977) in which the retention interval occurred either after the two stimuli to be compared had both been presented or between the first and the second stimulus. For both groups, the stimuli were red and green hues and the retention test consisted of a subsequent choice between the left and right side keys, with one choice reinforced if the two hues were identical and the other reinforced if they were different. Since reinforcement was possible on every trial, trial outcomes were essentially nondifferential with respect to the stimulus pairs in both groups. Nonetheless, Pontecorvo found better retention in the group for which the stimuli of each pair appeared in quick succession prior to the delay than when they were separated by the delay interval. This result, he argued, indicated that response intentions contributed to the retention differences: birds in the former but not the latter group could presumably anticipate early in the trial which side-key response to make after the retention interval. His conclusion was also supported by an analysis of intertrial interference, which showed that in the former group, the choice response birds made on the preceding trial was a major source of proactive interference (Wright, Urcuioli, \& Sands, 1986).

However, another interpretation of Pontecorvo's (1985) results is that birds in the group that saw both stimuli of a pair presented together at the beginning of a trial simply coded whether or not the first stimulus had changed. If remembering whether or not a stimulus has just changed in color is easier than remembering a specific color, this would account for Pontecorvo's results.

In any event, the present experiments clearly show that in a go/no-go procedure like that used by Honig and Wasserman (1981), response anticipations, if present, do not facilitate memory performances. By contrast, differential outcome expectancies appear to contribute substantially to the DSD-DCD difference. Future research can provide additional confirmation for this conclusion by showing that those presumed expectancies are sufficient to mediate transfer of performance across tasks.

\section{REFERENCES}

BLough, D. S. (1959). Delayed matching in the pigeon. Journal of the Experimental Analysis of Behavior, 2, 151-160.

Brodigan, D. L., \& Peterson, G. B. (1976). Two-choice conditional discrimination performance of pigeons as a function of reward expectancy, prechoice delay, and domesticity. Animal Learning \& Behavior, 4, 121-124.

Carlson, J. G., \& Wielkiewicz, R. M. (1976). Mediators of the ef fects of magnitude of reinforcement. Learning \& Motivation, 7. 184-196.

Carter, D. E., \& Eckerman, D. A. (1975). Symbolic matching by pigeons: Rate of learning complex discriminations predicted from simple discriminations. Science, 187, 662-664.

Cohen, J. S., Galgan, R., \& Fuerst, D. (1986). Retrospective and prospective short-term memory in delayed response tasks in rats. Animal Learning \& Behavior, 14, 38-50.

Delong, R. E., \& Wasserman, E. A. (1981). Effects of differential reinforcement expectancies on successive matching-to-sample perfor- 
mance in pigeons. Journal of Experimental Psychology: Animal Behavior Processes, 7, 394-412.

Hogan, D. E., Pace, G. M., \& Zentall, T. R. (1983). Control of pigeon's matching-to-sample performances by differential sample response requirements. American Journal of Psychology, 96, 37-49.

HoNIG, W. K. (1978). Studies of working memory in the pigeon. In S. H. Hulse, H. Fowler, \& W. K. Honig (Eds.), Cognitive processes in animal behavior (pp. 211-248). Hillsdale, NJ: Erlbaum.

HoNIG, W. K., \& DoDD, P. W. D. (1983). Delayed discriminations in the pigeon: The role of within-trial location of conditional cues. Animal Learning \& Behavior, 11, 1-9.

HonIG, W. K., \& DODD, P. W. D. (1986). Anticipation and intention in working memory. In D. F. Kendrick, M. E. Rilling, \& M. R. Denny (Eds.), Theories of animal memory (pp. 77-100). Hillsdale, NJ: Erlbaum.

Honig, W. K., Matheson, W. R., \& Dodd, P. W. D. (1984). Outcome expectancies as mediators for discriminative responding. Canadian Journal of Psychology, 38, 196-217.

Honig, W. K., \& Thompson, R. K. R. (1982). Retrospective and prospective processing in animal working memory. In G. H. Bower (Ed.), The psychology of learning and motivation: Advances in research and theory (Vol. 16, pp. 239-283). New York: Academic Press.

Honig, W. K. , \& Wasserman, E. A. (1981). Performance of pigeons on delayed simple and delayed conditional discriminations under equivalent training procedures. Learning \& Motivation, 12, 149-170.

Peterson, G. B. (1984). How expectancies guide behavior. In H. L. Roitblat, T. G. Bever, \& H. S. Terrace (Eds.), Animal cognition (pp. 135-147). Hillsdale, NJ: Erlbaum.

Pontecorvo, M. J. (1985). Memory for a stimulus versus anticipation of a response: Contrasting effects of proactive interference in two delayed comparison tasks. Animal Learning \& Behavior, 13, 355-364.

RODGER, R. S. (1975a). The number of non-zero, post hoc contrasts from ANOVA and error rate. I. British Journal of Mathematical \& Statistical Psychology, 28, 71-78.

RODGER, R. S. (1975b). Setting rejection rate for contrasts selected post hoc when some nulls are false. British Journal of Mathematical \& Statistical Psychology, 28, 214-232.
SHIMP, C. P., \& MoffitT, M. (1977). Short-term memory in the pigeon: Delayed-pair comparison procedures and some results. Journal of the Experimental Analysis of Behavior, 28, 13-25.

Trapold, M. A. (1970). Are expectancies based upon different positive reinforcing events discriminably different? Learning \& Motivation, 1, 129-140.

URCUIOLI, P. J. (1985). On the role of differential sample behaviors in matching-to-sample. Journal of Experimental Psychology: Animal Behavior Processes, 11, 502-519.

Urcuioli, P. J., \& Honig, W. K. (1980). Control of choice in conditional discriminations by sample-specific behaviors. Journal of $E x$ perimental Psychology: Animal Behavior Processes, 6, 251-277.

Weisman, R. G., Bruce, R., \& Beninger, R. J. (1987). Simple and conditional discrimination in rats: The effects of delays and scopolamine. Learning \& Motivation, 18, 274-287.

Wright, A. A., Urcuioli, P. J., \& SANds, S. F. (1986). Proactive interference in animal memory. In D. F. Kendrick, M. E. Rilling, \& M. R. Denny (Eds.), Theories of animal memory (pp. 101-125). Hillsdale, NJ: Erlbaum.

Zentall, T. R., Hogan, D. E., Howard, M. M., \& Moore, B. S. (1978). Delayed matching in the pigeon: Effect on performance of sample-specific observing responses and differential delay behavior. Learning \& Motivation, 9, 202-218.

\section{NOTE}

1. It is not clear why Groups SD and CD responded less often on average on go trials than did Groups $\mathrm{SE}$ and $\mathrm{CE}$. One possibility might be that reinforcement of not responding on half of the trials (viz. on no-go trials) in the former two groups also depressed their responding on go trials.

(Manuscript received May 15, 1989; revision accepted for publication August $22,1989$. 